\begin{tabular}{c} 
International Journal of Engineering \& Technology, 7 (3) (2018) 1181-1184 \\
International Journal of Engineering \& Technology \\
SPC \\
Website: $\begin{array}{c}\text { www.sciencepubco.com/index.php/IJET } \\
\text { doi: } 10.14419 / \text { ijet.v7i3.13097 } \\
\text { Research paper }\end{array}$ \\
\hline
\end{tabular}

\title{
Improving MIMO system throughput using power transmission scheduling
}

\author{
Khaleelahmed SK ${ }^{1}$ *, Venkateswararao $\mathrm{N}^{2}$, Varshasree $\mathrm{KN}^{1}$, P.V. Naidu ${ }^{1}$ \\ ${ }^{1}$ Velagapudi Ramakrishna Siddhartha Engineering College, Vijayawada-520007, India \\ ${ }^{2}$ Bapatla Engineering College, Bapatla-522101, India \\ *Corresponding author E-mail: khaleelto786@gmail.com
}

\begin{abstract}
The main idea of this paper is to select the best power allocation throughput model by simulating the different power allocation theoretical modules in MIMO arbitrary multipath environment to address the effects of channel parameters on throughput. In general, power allocation techniques are used for minimizing the overall Bit Error Rate (BER). In this process, the channel estimation is usually done at the receiver by accessing the Channel State Information (CSI). The optimized system can be designed with respect to channel parameters so that it can be suitable for transmitter side during power allocation. The simulation analysis is carried out in NI LabVIEW and it is observed from the studies that the throughput results are as a function of received power. Under static system parameters, the relative throughput of the water filling (WF) power allocation model is found to be high efficient $15.74 \%$ when it is compared with the open loop zero-forcing (ZF) and it is $4.45 \%$ with respect to inverse singular value decomposition (SVD) equal power allocation models.
\end{abstract}

Keywords: Multiple-Input-Multiple-Output (MIMO); Bit-Error-Rate (BER), Channel-State-Information (CSI); Water-Filling (WF), Zero-Forcing (ZF); Singular-Value-Decomposition (SVD.

\section{Introduction}

MIMO is the approach of proliferating the capacity of radio stream using multiple transmitters and multiple receivers through multipath propagation. Better communication achieved only when channel status is good in the multipath propagation environment [1]. Propelled by the demands of the MIMO users, different power allocation schemes were used in order to boost the throughput and make the system energy efficient [2]. Here throughput is an important metric which conveys the net amount of data delivered to the receiver in the MIMO system from transmitter [3].

In SVD systems [4], the power to be transmitted is equally distributed within the transmitter antennas which is familiar as SVD equal power allocation technique, by which mean energy consumption is dropped but error probability of transmission will be high compared with the other techniques. Hence, in terms of fixed transmission rate and link reliability SVD systems acts well. So, SVD Inverse power allocation method is introduced in [5]. In [6] to allocate more power to weaker channel and allocate less power to the better channel of the MIMO system was discussed. In inverse power allocation, consideration starts with the inverse of parameter matrix [7]. Comparing with SVD systems Zero Forcing method of power allocation systems decoupling complexity will be high, as it forms an open loop with the addition of weights during transmission [8]. The MIMO decoupling complexity is directly proportional to the difficultness in designing the ZF precoders at transmitter side [9].

Water filling [10] power allocation technique is one of the most popular technique in wireless communications. By this method more power is allocated for each transmission stream with high gain for improvement in sum data rate of all channels. This approach [10] reduces the computational complexity of transmission compared with the other systems [11]. Because additional interference constraints in the system, the water-filling algorithms are always performed iteratively to solve the power allocation problems in the system along with the system complexity [12]-[13].

In this paper, we are simulating a direct power allocation method for achieving better throughput with optimal power allocation in Physical Downlink Shared Channel . The rest of the paper is organized as follows. The $2 \times 2$ MIMO system model is present in section 2 and theoretical throughput model is discussed in Section 3 and in Section 4 the numerical results are given.

\section{System model}

A conventional model of alamouti STBC MIMO communication system is designed including two transmitter antennas and two receiver antennas [16]. The data from the source is mapped using symbol mapper. Those symbols are passed through M-QAM modulator. The modulated symbols are sequentially disseminated through alamouti STBC Encoder. In each substream, modulated bits are mapped into signal waveforms which are directly transmitted through transmitter antennas to the receiver side. In Fig.1, the data is transmitted to the receiver side through a wireless channel. The channel consists of Rayleigh fading and introduces Additive White Gaussian Noise (AWGN). At the receiver side, the received data directly sent into alamouti STBC Decoder which incorporates equalizer aids to predict the channel state estimation (CSI) and noise cancellations [5]. 


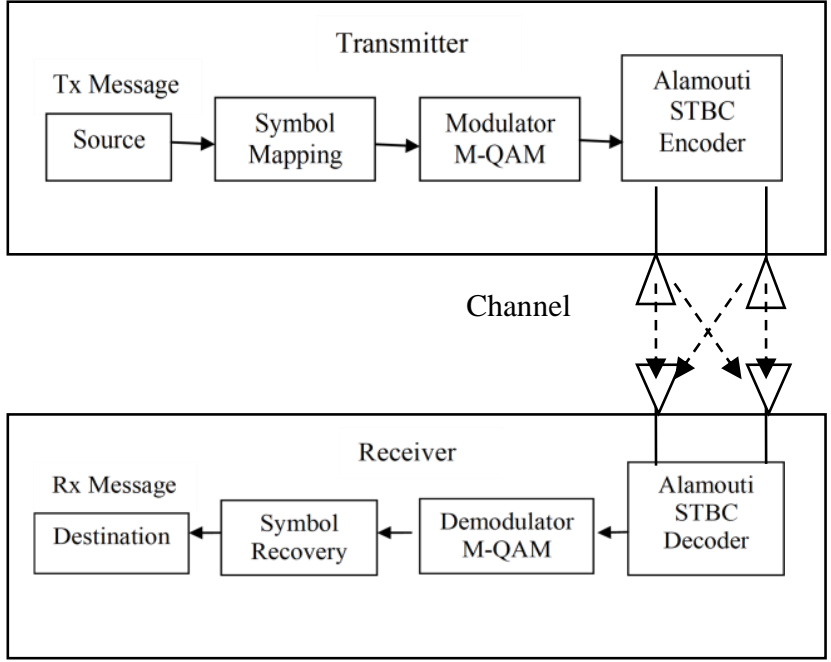

Fig. 1: Generalized Block Diagram of 2 x 2 MIMO System Model [16]

In equalizer, a channel estimator, combiner, and Maximum likelihood detector assist to combine the signals for maximum likelihood detection, and error probability calculations. Then, the combined signals are demodulated followed by the symbol recover for data recovery.

\section{Throughput models}

In a flat fading channel, A MIMO system of order $\mathrm{m} \mathrm{x} \mathrm{n}$ is formulated. It is illustrated as

$$
y=H x+n
$$

Where $\mathrm{y}$ is receiver signal vector, $\mathrm{x}$ is transmitter signal vector, $\mathrm{n}$ is noise vector with unconventional homogeneously distributed Gaussian variable and $\mathrm{H}$ represents system matrix [14]. Through channel evaluation and tracing, the MIMO systems receiver part recoup the channel state information (CSI) of the channel. In Time Division Duplexing (TDD) systems, the transmitter will rake in CSI by depicting on channel reciprocity (ceasing that the channel will be synonymous with conjoining uplink and downlink channels). When Channel State Information available at both margins, then SVD techniques are used. Let the SVD of $\mathrm{H}$ is

$$
H=U \Lambda V^{H}
$$

Where $\mathrm{V}$ and $\mathrm{U}$ are the unitary matrices, and $\Lambda$ is a diagonal matrix consisting of the singular values of $\mathrm{H}$. Then by accumulating the signal vector $\mathrm{S}$ with $\mathrm{VP}$, the precoding, and power allocation will be done i.e., $\mathrm{x}=$ VPS where $\mathrm{V}$ is unitary matrix obtained from above equation, $\mathrm{P}$ is a diagonal matrix whose elements refers the allocated power to each stream. In inverse power allocation strategy, large-scale power is added for a weaker stream and less power is added to the stronger stream in the system. Then the power allocation vector is represented as $P=\Lambda^{-1} \mu$. Where the parameter $\mu$ is a constant making sure that the total power is the same before and after the precoding and power allocation VP as F.

$N_{t}=\operatorname{tr}\left[F^{H} F\right]=\operatorname{tr}\left[P^{H} V^{H} V P\right]=\operatorname{tr}\left[P^{2}\right]=\mu^{2} \operatorname{tr}\left[\wedge^{-2}\right]$

Here $\mathrm{P}$ is the allocated power vector, $\mu$ is given by

$\mu=\sqrt{N_{t} / \operatorname{tr}\left(\Lambda^{-2}\right)}$

Where $N_{t}$ is a number of antennas at the transmitter side. It is incited to make the probability of distribution $(\mathrm{PoD})$ better in every stream as it balances $\mathrm{PoD}$ of every channel through the proper power supply. Power is predefined and the power is divided equal- ly among all the channels of MIMO system in SVD Equal power allocation method. It is given by

$\mathrm{p}=\mathrm{P} / \mathrm{n}$

Where $\mathrm{p}$ is power assigned per a channel, $\mathrm{P}$ is total power, $\mathrm{n}=$ number of channels. Important thing is, these SVD strategies, apt when channel state information is clearly notable at both ends of MIMO system [6].

When channel state information is at hand on only one side, then Zero Forcing method will be suitable. Zero Forcing is a procedure to compensate the delays in antennas of MIMO system, by adding additional weights to it so the weighted sum produces zero deviation in the frequency domain. Power allocation matrix through zero forcing method will be given as

$\Lambda^{i}=1 / \sqrt{\left(H_{i}^{H} H_{i}\right)^{T}}$

Where $\Lambda^{i}$ is the power allocation matrix, $\mathrm{H}$ is the MIMO system matrix [8], equation (6) is used to decouple the MIMO system for the power constraint of channels. In general, it is very difficult to allocate a power individual subchannels in the OFDM-based MIMO Systems [12]. This power allocation problem cannot be solved simply by applying the traditional water-filling method.

The power allocation is expressed as

$$
p_{\text {total }}=\sum_{n=0}^{N-1}\left(p_{\text {Level }}-\frac{N_{o}}{\left(h_{n}\right)^{2}}\right)
$$

Where $P_{n}$ is power allocated for the $n$ 'th channel, PLevel is power level that required to fill, $N_{o}$ is noise in the channel and $h_{n}$ is specified channel parameters. The total power is given in (7), because additional interference constraints must be considered in MIMO Systems. The water-filling algorithms are always performed iteratively to solve the power allocation problem through binary search method [11], [12].The relative throughput of the total communication system will be given using the probability distribution of the model. Hence the relative throughput $\left(T_{p u}\right)$ will be

$T_{P_{u t}}(\bar{\gamma})=T_{P_{u, \max }}\left(1-F\left(\gamma_{t h} / \frac{-}{\gamma}\right)\right)$

Where $\gamma$ is instantaneous SNR, $\gamma_{t h}$ is threshold SNR, $T_{P u, \text { max }}$ indicates maximum data rate and $\mathrm{F}$ denotes the cumulative distribution function. For Rayleigh fading channels, the probability and cumulative distribution functions are exponential functions [15].

\section{Simulations}

The NI LabVIEW simulation model for $2 \times 2$ MIMO with Alamouti STBC using M-QAM in Rayleigh fading with AWGN channel is performed in the range of received power $-90 \mathrm{dBm}$ to $45 \mathrm{dBm}$.In Fig.2, the total number of symbols generated using the Multi-Level Quadrature Amplitude Modulation (M-QAM) is 1500 and each symbol consists of 8 samples. A number of symbols in the modulation constellation is 256, shaped using Root Raised Cosine pulse shaping filter. The ratio of Energy per bit to the noise power spectral density is assumed to be 50 and channel filter length for symbols is taken as 8 in low pass channel filter. Training length of symbols and Equalizer length of symbols are 500 and 48 at operational frequency assumed to $915 \mathrm{MHz}$, the system bandwidth is $75 \mathrm{kHz}$, which is lower than the coherence bandwidth In Fig. 3, the threshold value of power $-65.44 \mathrm{dBm}$ is achieved from the measurement of $41.57 \%$ relative throughput for SVD Inverse power allocation whereas for water filling is $42.77 \%$ relative throughput observed. For SVD Equal power allocation 36.15\% relative throughput noticed whereas for Zero Forcing method of power allocation $33.30 \%$ relative throughput is observed. 


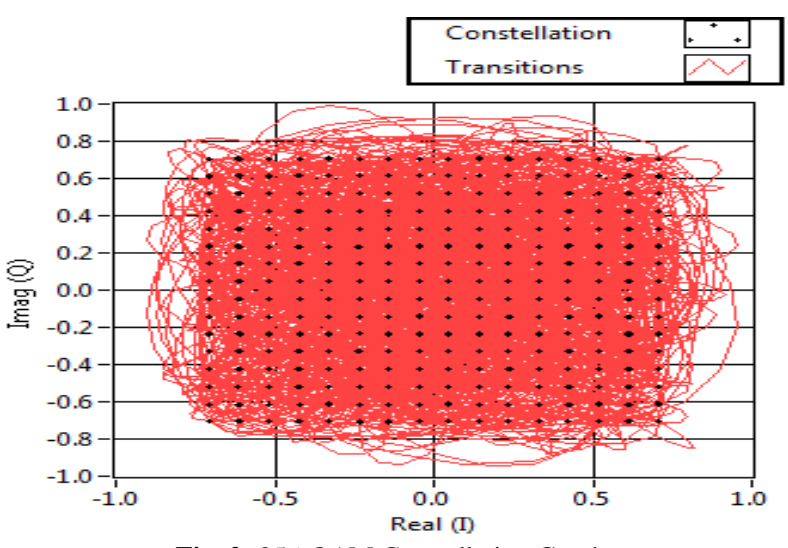

Fig. 2: 256-QAM Constellation Graph.

From the Fig.4, it is observed that SVD MIMO system lacking the Inverse scheme, is inferior to the open loop ZF method of power allocation.

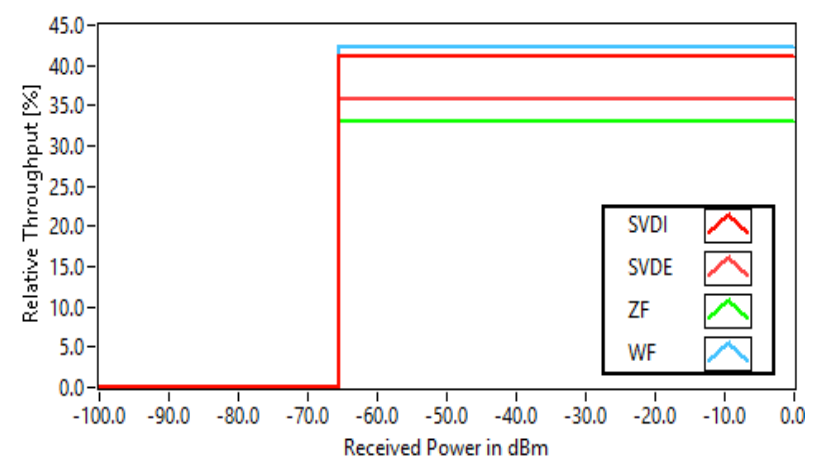

Fig. 3: Relative Throughput of Power Allocation Methods at Power Level $65.44 \mathrm{dbm}$.

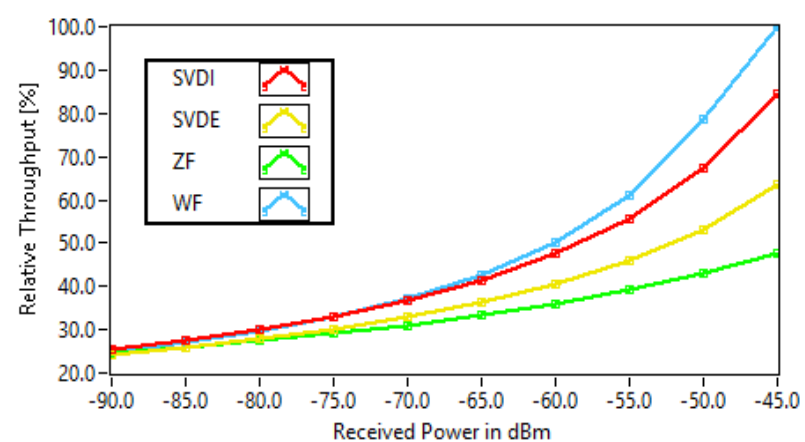

Fig. 4: Relative Throughput of Power Allocation Methods in Power Range (-90dbm to $-45 \mathrm{dbm})$.

The main purpose of degradation of SVD is for a worthy channel, SVD performs better than the ZF method whereas, for an unsatisfactory channel, ZF will be more suitable than SVD, From the graphical representation, it can be concluded that for lower power levels all power allocation techniques work similar and as power increase the variation between relative throughputs of power allocation techniques observed. Finally, Water Filling method of power allocation works better than the other power allocation techniques.

Table 1: Average Relative Throughput

\begin{tabular}{ll}
\hline $\begin{array}{l}\text { Power Allocation Tech- } \\
\text { nique Used }\end{array}$ & $\begin{array}{l}\text { Average Relative Throughput in power range } \\
(-90 \mathrm{dBm} \text { to }-45 \mathrm{dBm})\end{array}$ \\
\hline Zero Forcing & 33.65 \\
SVD Equal & 37.96 \\
SVD Inverse & 44.95 \\
Water Filling & 49.39 \\
\hline
\end{tabular}

\section{Conclusion}

The throughput of different power allocation methods on MIMO systems is designed for NI LabVIEW software. The water filling method on 2x2 MIMO system is tested and compared with other techniques. SVD-based MIMO with the inverse power allocation is approximately $7 \mathrm{~dB}$ to $10 \mathrm{~dB}$ better than SVD Equal and Zeroforcing power allocation methods, water-filling method nearly $5 \mathrm{~dB}$ better than the closed loop SVD based MIMO system.

\section{References}

[1] D. Hassan, Y. Kirsal \& S. Redif, "Channel capacity improvement for cooperative MIMO wireless sensor networks via adaptive MIMO-SVD" , HONET-ICT, 2016, pp. 49-53, https://doi.org/10.1109/HONET.2016.7753449.

[2] Mittu, Sangeeta, Avinash Manu \& Aravindan, "Optimal Power Allocation Techniques using SVD for MIMO-OFDM Multimedia Mobile Networks", International Journal of Engineering Trends and Technology (IJETT), Vol.26, No.5,2015, pp.239-245. https://doi.org/10.14445/22315381/IJETT-V26P242.

[3] J. Zhang \& J. S. Lehnert, "Throughput Optimal Precoding and Rate Allocation for MISO Systems with Noisy Feedback Channels", IEEE Transactions on Information Theory, Vol. 54, No.5, 2008 pp.2139-2155, https://doi.org/10.1109/TIT.2008.920342.

[4] J. Choi, J. Park \& B. L. Evans, "Spectral Efficiency Bounds for Interference-Limited SVD-MIMO Cellular Communication Systems", IEEE Wireless Communications Letters, Vol. 6,No. 1, 2017,pp. 4649, https://doi.org/10.1109/LWC.2016.2629474

[5] F. Ito, F. Uzawa, K. Mitsuyama \& N. Iai, "Performance evaluation of TDD-SVD-MIMO system with feedback delays", International Symposium on Antennas and Propagation (ISAP), 2017, https://doi.org/10.1109/ISANP.2017.8228736.

[6] X. Chen, B. P. Einarsson \& P. S. Kildal, "Improved MIMO Throughput With Inverse Power Allocation Study Using USRP Measurement in Reverberation Chamber", IEEE Antennas and Wireless Propagation Letters, Vol. 13, 2014, pp. 1494-1496, https://doi.org/10.1109/LAWP.2014.2342217.

[7] W. P. Hunek, "New SVD-based matrix H-inverse vs. minimumenergy perfect control design for state-space LTI MIMO systems", 20th International Conference on System Theory Control and Computing (ICSTCC), 2016, https://doi.org/10.1109/ICSTCC.2016.7790633.

[8] X. Chen, P. S. Kildal \& M. Gustafsson, "Characterization of Implemented Algorithm for MIMO Spatial Multiplexing in Reverberation Chamber", IEEE Transactions on Antennas and Propagation, Vol. 61, No. 8, 2013, pp. 4400-4404, https://doi.org/10.1109/TAP.2013.2259459.

[9] SK. Mohammed \& E. G. Larsson, "Improving the Performance of the Zero-Forcing Multiuser MISO Downlink Precoder Through User Grouping", IEEE Transactions on Wireless Communications, Vol. 15, No. 2, 2016, pp. 811-826, https://doi.org/10.1109/TWC.2015.2478878.

[10] P. He, L. Zhao \& B. Venkatesh, "Novel Water Filling for Maximum Throughput of Power Grid, MIMO, and Energy Harvesting Coexisting System With Mixed Constraints", IEEE Transactions on Communications, Vol. 65, No. 2, 2017, pp. 827-838, https://doi.org/10.1109/TCOMM.2016.2638907.

[11] L. Zhao, Y. Wang \& P. Chargé, "Efficient iterative water-filling power allocation method in MU-MIMO broadcast channels", Military Communications and Information Systems Conference (MCC), 2013.

[12] Qi, Qilin \& Yang, Yaoqing Lamar, " An Efficient Water-Filling Algorithm for Power Allocation in OFDM-Based Cognitive Radio Systems", International Conference on Systems and Infor-matics (ICSAI2012), 2012, $\quad$ Pages: $2069 \quad$ - 2073, https://doi.org/10.1109/ICSAI.2012.6223460.

[13] Chin-Wei Hsu, Ming-Fu Tang \& Borching Su," Power allocation for downlink path based precoding in multiuser FDD massive systems without CSI feedback", IEEE 50th Asilomar Conference on Signals, Systems and Computers, 2016, pp: 198 -202, https://doi.org/10.1109/ACSSC.2016.7869023.

[14] Chen, Xiaoming, Kildal, Per-Simon \& Gustafsson Mattias, " Comparing throughputs of $2 \times 2$ spatial multiplexing MIMO systems with and without CSI at the transmit side in rich isotropic multipath envirments ", 2014, pp.3493-3494, https://doi.org/10.1109/EuCAP.2014.6902582. 
[15] P.-S. Kildal et al, "Threshold receiver model for throughput of wireless devices with MIMO and frequency diversity measured in reverberation chamber", IEEE Antennas Wireless Propag.Lett, Vol.10, 2011, pp. 1201-1204 https://doi.org/10.1109/LAWP.2011.2172909.

[16] V. D. Nehete \& K. Ziri-Castro, "A study of 2 × 2 MIMO system with Alamouti STBC in SDR with LabVIEW and NI-USRP 2953R", IEEE 85th Vehicular Technology Conference (VTC 2017), 2017. 